\title{
CRÓNICA ITALIANA: EN EL FILO DE LAS DECISIONES POLÍTICAS CAPITALES. ELECCIONES GENERALES Y REFERÉNDUM CONSTITUCIONAL
}

\author{
CARLOS ORTEGA SANTIAGO \\ Profesor de Derecho Constitucional \\ Universidad de Valladolid
}

SUMARIO

I. Algo debe cambiar... para que todo siga igual.

II. Eppur si muove...

"El que desciende al abismo de la Muerte y sube por el Árbol de la Vida..., llega a la Ciudad de lo Posible, desde la cual se contempla el Todo y se deciden las Opciones.".

(ITALO CALVINO, El castillo de los destinos cruzados)

\section{ALGO DEBE CAMBIAR... PARA QUE TODO SIGA IGUAL...}

En la política italiana han tenido lugar en los últimos meses acontecimientos con relevancia constitucional de aquéllos que pueden determinar el futuro de una sociedad y del Estado que la encarna. Además, las decisiones públicas adoptadas en este tiempo han podido ser de un signo o de otro, en sentidos radicalmente diferentes, por escasos márgenes de ventaja numérica, esos que derivan de la dimensión aritmética del principio mayoritario.

Que esto haya podido ocurrir, esto es, que el sistema electoral previsto en la ley, o que la forma de gobierno y de organización territorial previstas en la Constitución, se hayan convertido en decisiones de la mayoría y no hayan contado con el consenso mínimo de la oposición, se debe sin ninguna duda a la crisis de la que aún no se ha recuperado el sistema político italiano, donde viene siendo harto improbable que se delimiten espacios de confluencia y de integración entre los diferentes polos del espectro partidista, incluso cuando se 
trata de las decisiones fundamentales para una comunidad política (cómo se elige a los que ejercerán el poder, cómo se organizará éste a nivel nacional, cómo se estructurará territorialmente).

En último extremo, puede decirse que ha sido el cuerpo electoral, los ciudadanos votantes en las elecciones y en el referéndum constitucional, los que han reintegrado el sistema político a su cauce natural: La Constitución de 1948, que fue fruto del amplio consenso constituyente que reestructuró al país después de la II Guerra Mundial, y que ha sido resultado también del consenso que se forja en torno a una Constitución a través de su efectiva vigencia (realizada la norma fundamental por el legislador y, de manera esencial, por la jurisdicción constitucional).

Pero se hace necesario acotar los temas conflictivos y complejos de la vida política italiana del último año para intentar arrojar una mínima luz sobre la maraña de reformas normativas tramitadas en el Parlamento de este país, y sobre los principios que las han sustentado y los objetivos (más o menos legitimos) que las han animado. Sin duda, la reforma de las leyes electorales que disciplinan la elección de la Cámara de Diputados y del Senado, y la propuesta de reforma de más de cincuenta artículos de la Constitución, propuesta finalmente no ratificada en referéndum, son los dos hitos fundamentales en los que se ha manifestado el desencuentro político entre las fuerzas parlamentarias de la mayoría y de la oposición.

En cuanto a las reformas electorales que modifican los dos textos normativos que disciplinan la elección de las cámaras legislativas nacionales, un amplio sector de la doctrina constitucional se ha pronunciado por la inconstitucionalidad de dichas modificaciones. Dejando a un lado los cálculos políticos que parecen haber informado la reforma, y que se habrían encaminado a facilitar la consecución de una nueva mayoría parlamentaria a favor del Polo y del Presidente del Consejo Berlusconi en las elecciones de 2006, las dudas que suscita la nueva disciplina electoral acerca de su adecuación a la Constitución son numerosas.

En primer lugar, la ley de reforma ha optado por retornar al sistema electoral proporcional para transformar los votos de los ciudadanos en escaños a la Cámara de Diputados y al Senado. Se abandona, así, el sistema electoral mayoritario, y se retorna al viejo sistema proporcional, que en Italia no puede dejar de vincularse, desde la entrada en vigor de la Constitución republicana, a los gobiernos de amplias coaliciones de partidos, a la inestabilidad del gobierno, y a la partidocracia.

En este sentido, no puede obviarse que el cambio al sistema electoral mayoritario fue fruto de un impulso popular que condujo a la solicitud de un referéndum derogador de la disciplina electoral proporcional, y a su sustitución por un sistema mixto con prevalencia mayoritaria. Como consecuencia del referéndum de 1993 que derogó partes significativas de la ley electoral del Senado, el Parlamento italiano aprobó las leyes n. ${ }^{\text {os }} 276$ y 277 de 1993 , que sellaban definitivamente el final del anterior sistema de partidos con la introducción de un sistema mayoritario uninominal con correctivo proporcional en ambas Cámaras. 
Ciertamente, la experiencia de los años de vigencia de este sistema arroja algunas sombras en cuanto a su virtualidad para reducir la complejidad multipartidista y su posterior traslación a las Cámaras legislativas, cuya muestra más evidente es la existencia de una pluralidad de partidos en cada uno de los polos resultantes de las coaliciones necesarias para vencer en los distritos uninominales y formar, así, una mayoría de gobierno. De esta forma, en el Polo delle Libertà se integran en torno a cinco partidos, con preeminencia de Forza Italia, mientras que en la Unione son cerca de nueve las formaciones políticas, de desigual importancia, con preeminencia de L'Ulivo.

En los comienzos del sistema mayoritario pudo pensarse, incluso, que nada había cambiado en cuanto a la posible eliminación de la partidocracia, de las cuotas de poder en el gobierno (empezando por las vicepresidencias y los ministerios, y concluyendo por el reparto de los altos cargos), y de la inestabilidad parlamentaria de las mayorías; como se demostró en la cortísima duración del primer gobierno Berlusconi, después de que la Lega Nord abandonase la coalición.

Pero también es cierto que alguna mejora había procurado la nueva legislación electoral respecto del viejo sistema político, ya que bajo su vigencia se ha producido la primera crisis de gobierno exteriorizada en el ámbito parlamentario y concretada a través de mecanismos específicamente dispuestos en la Constitución para medir la confianza del ejecutivo en el ámbito legislativo [nos referimos a la primera caída de un gobierno como consecuencia de una moción de censura parlamentaria (el primer gobierno Prodi, 1998)]; y por primera vez, también, un gobierno y su mayoría parlamentaria han subsistido durante los cinco años de la legislatura [el segundo gobierno Berlusconi (2001-2006)]. Sin olvidar que a partir de la instauración del mayoritario se ha producido una auténtica alternancia en el poder, al uso de las democracias (parlamentarias y presidenciales) actuales.

Esta realidad histórica de la instauración del sistema electoral mayoritario a partir de la decisión popular afirmada en referéndum venía a demostrar, además, la superación de algunos planteamientos doctrinales que habían sostenido en su momento la existencia de una opción del constituyente italiano por el sistema electoral proporcional $^{1}$; vinculado éste, a su vez, a la falta de una homogeneidad política suficiente en la sociedad italiana capaz de construir un sistema bipartidista, al menos en cuanto a la alternancia en el ejercicio del poder.

En realidad, ésta ha sido la duda que no había sido disipada aún durante la vigencia del mayoritario en los procesos electorales nacionales, si la evolución del sistema de partidos con la aplicación de este sistema electoral acabaría reduciendo el número de formaciones políticas con representación parlamentaria, y lo que es más importante todavía, el peso de cada una de ellas en la construcción de una mayoría de gobierno.

1 LAVAGNA, C., "Il sistema elettorale nella Costituzione italiana”, en Revista trimestrale di diritto pubblico, n. ${ }^{\circ} 3,1952$. 
La reforma electoral aprobada con la ley n. ${ }^{\circ} 270$ de 2005 , pocos meses antes de la celebración de las elecciones generales de 2006, ha impedido comprobar en este proceso electoral esa virtualidad del sistema mayoritario. Precisamente la referida ley se ha aprobado, en teoría, con el objeto de paliar las deficiencias del mayoritario, con la finalidad de permitir la creación de mayorías estables de gobierno en ambas ramas del Parlamento. Evidentemente, eso no parece muy razonable si tenemos en cuenta que la ley establece el sistema electoral proporcional para la elección de la Cámara de Diputados y del Senado, y además instaura el sistema del resto mayor que, como es sabido, no se caracteriza por premiar a las listas más votadas, sino por tender a una mayor proporcionalidad en el reparto de escaños, en beneficio de las formaciones políticas menos votadas.

De ahí que nos encontremos, con toda evidencia, en una decisión de la mayoría parlamentaria que sustentaba al gobierno Berlusconi de pura coyuntura y estrategia política; no tiene sentido intentar afianzar mayorías estables a partir de un sistema proporcional puro. Por ello, esta proporcionalidad se pretende corregir en la propia ley a través de una institución, el premio de mayoría a favor del partido o de la coalición de partidos más votada, que suscita fuertes dudas de constitucionalidad y que, desde una perspectiva de análisis de los procesos electorales como a la que induce nuestro ordenamiento, es difícil de entender. Pero vayamos por partes.

Por lo que se refiere a la Cámara de Diputados, la ley establece la circunscripción electoral provincial, en el caso del Senado la circunscripción es la región. Los partidos políticos presentan sus propias candidaturas en cada una de las circunscripciones y, al mismo tiempo, han de declarar si se vinculan a una determinada coalición de partidos, los cuales comparten un mismo programa electoral y un único jefe de la coalición. A su vez, los partidos pueden concurrir a las elecciones en solitario, sin coaligarse, designando también a una persona como líder de la formación política, aunque, como diremos después, el sistema electoral obliga a los partidos a tender a coaligarse si quieren alcanzar representación parlamentaria y contar en la mayoría de gobierno.

Ese programa y ese liderazgo deben ser consignados en la fase de la presentación de candidaturas, fundamentalmente con el objeto de que los electores puedan conocer con qué candidato a la presidencia del gobierno y con qué programa se vincula cada partido. Sin embargo, estas previsiones no dejan de suponer un simple vínculo político, y sólo podrían cobrar pleno sentido jurídico con otra disciplina constitucional de la forma de gobierno, en concreto la que se preveía en la propuesta de reforma de la Constitución que finalmente no ha prosperado y sobre la que volveremos después.

En efecto, es claro que esta regulación legal pretende fortalecer y hacer especialmente visible la figura del líder de cada coalición de partidos en el proceso electoral y derivar de ahí, correlativamente, una legitimación política y una elección semidirecta del jefe del gobierno por parte de los ciudadanos. Pero, como la propia ley no puede dejar de prever, esta regulación del liderazgo de las coaliciones electorales y de los partidos ha de hacerse sin perjuicio de las 
prerrogativas que corresponden al Presidente de la República ex art. 92.2 de la Constitución italiana vigente; precepto que otorga a esta magistratura cierto margen de maniobra a la hora de designar al presidente del gobierno, de acuerdo con los resultados electorales y con las posiciones de cada partido para definir una mayoría parlamentaria.

En otras palabras, bajo la vigente Constitución el Presidente de la República no se encuentra obligado a nombrar jefe de gobierno al candidato de la coalición que haya obtenido más escaños; aunque no puede considerarse que la decisión del Presidente de la República sea enteramente libre en un sistema parlamentario como el italiano, puesto que sí viene vinculado por las posiciones de los partidos en cuanto a su apoyo a uno o a otro candidato, a partir de las diferentes mayorías parlamentarias que de ahí puedan derivarse.

Volviendo al sistema electoral previsto en la Ley 270 de 2005, en relación con la Cámara de Diputados, hay que tener en cuenta que el reparto de escaños que corresponde a cada coalición de partidos se hace en un primer momento a nivel nacional; en este ámbito opera, además, el llamado premio de mayoría. Para determinar cuantos escaños corresponden a cada coalición o a cada partido, se toman en consideración los votos que han recibido en cada circunscripción electoral las listas presentadas por cada partido, ya se integre en una coalición ya concurra en solitario.

A partir de esa cifra, la Ley determina que entran en el reparto de escaños sólo las coaliciones que hayan obtenido en el ámbito nacional al menos el $10 \%$ de los votos válidos emitidos y siempre que, al mismo tiempo, las listas presentadas por uno de los partidos coaligados hayan obtenido en el ámbito nacional al menos el 2\% de los votos válidos (con la alternativa a este último requisito de que un partido coaligado representativo de minorías lingüísticas obtenga el $20 \%$ de los votos en una sola circunscripción). Dentro de cada coalición que supere las referidas barreras legales se produce un ulterior reparto, al que sólo acceden los partidos coaligados que hayan alcanzado el 2\% de los votos a nivel nacional; no obstante, el partido más votado de una coalición de entre los que no alcanzan el referido $2 \%$ entra también en el reparto de escaños.

Por lo tanto, los partidos que integran una coalición que no alcanza el referido $10 \%$ de votos no pueden entrar en el reparto de escaños como consecuencia de esta barrera legal; salvo que, como excepción que prevé la propia ley, las listas del partido hayan alcanzado al menos el $4 \%$ de votos válidos a nivel nacional, supuesto en el que entrarían en el reparto a título individual.

Esta previsión cobra pleno sentido si tenemos en cuenta que, a su vez, la ley establece una barrera legal del 4\% para entrar en el reparto de escaños respecto de aquellos partidos que no se hubieran coaligado. En cualquier caso, si se observa bien, aquí se pone de manifiesto uno de los tratos diferenciadores contenidos en la ley, de entre otros que han sido criticados fuertemente en sede doctrinal, con visos de carencia de razonabilidad, puesto que un partido puede entrar en el reparto de escaños con el $2 \%$ de los votos si se ha integrado en una coalición (o incluso sin alcanzar esa barrera legal, siendo el más votado de 
los que quedan en la coalición por debajo de ella, como se ha dicho), pero si no se ha coaligado necesita alcanzar el $4 \%$ de los votos.

La justificación a esta previsión normativa podría estar en promover la agregación entre partidos y evitar la fragmentación de la Cámara; pero esta virtualidad de la medida diferenciadora no puede garantizarse jurídicamente desde el momento en que la ley no prevé (ni puede prever, en el marco de la Constitución vigente) medidas vinculantes y sanciones jurídicas para aquellas formaciones políticas que se separen de su coalición una vez alcanzado el éxito electoral con la obtención de representación parlamentaria (así se deriva necesariamente de la prohibición de mandato imperativo sobre los parlamentarios prevista en el art. 67 de la Constitución italiana).

En definitiva, la ley no garantiza la subsistencia de una coalición (y de una mayoría parlamentaria) durante el transcurso de la legislatura, con la consiguiente posibilidad de que un partido se beneficie de barreras legales más reducidas por haberse coaligado, optando después por nuevas alianzas parlamentarias e, incluso, por coadyuvar a configurar una nueva mayoría parlamentaria de gobierno (ribaltone). Como se ha señalado por la doctrina, estas previsiones contenidas en la reforma electoral podían disponer de una cobertura constitucional suficiente en el proyecto de modificación constitucional que tramitaba el Parlamento italiano cuando se aprobó la ley electoral, ya que allí se preveía un vínculo de coalición sancionable jurídicamente; pero, evidentemente, ésa no era la disciplina constitucional de referencia cuando entró en vigor la ley n. ${ }^{\circ} 270$ de 2005 , y no puede serlo tampoco a partir de la no ratificación de la reforma en referéndum.

En este orden de cosas, también se ha afirmado la carencia de razonabilidad de las previsiones de la ley que regulan el premio mayoritario en beneficio de aquella coalición de partidos (o, con escasa probabilidad, para aquel partido) que haya obtenido más votos a nivel nacional en las elecciones a la Cámara de Diputados. Para garantizar la estabilidad de la mayoría parlamentaria que corresponde a esta coalición vencedora en los votos, la Ley prevé que se le asignarán automáticamente el $54 \%$ de los escaños de la Cámara (340 sobre 630), con independencia del porcentaje de votos obtenido, salvo que la cifra de votos conlleve un número mayor de escaños.

Ciertamente, cuanto mayor sea el número de partidos coaligados y menor el número de coaliciones, menor será también el desvío de la proporcionalidad que puede derivarse de este premio mayoritario; pero también es cierto que en un proceso electoral fuertemente bipolarizado, con dos coaliciones con posibilidades de entrar en el reparto de escaños, es más factible que una mínima diferencia porcentual de votos entre ambos polos (llegando al extremo de un único voto de diferencia), se traduzca en una diferencia más amplia en escaños.

El ejemplo elocuente de estas dos afirmaciones lo encontramos en la primera aplicación de la ley (elecciones legislativas de 2006), en la que una diferencia de votos del $0,1 \%$ se tradujo, como consecuencia del premio mayoritario, en una diferencia del 10,4\% en el número de escaños en la Cámara de Diputados a favor de la coalición Unione-Prodi frente a la coalición Casa delle 
Libertà-Berlusconi. Por otra parte, la desviación entre el porcentaje de escaños obtenido por la coalición vencedora (54\%) y su porcentaje de votos (49,8\%) presenta una desviación escasa, dada la fuerte agregación del voto en torno a dos coaliciones; desviación inferior incluso a la que se ha dado en algunas ocasiones en nuestro ordenamiento en las elecciones al Congreso de los Diputados.

Sin embargo, lo que ocurre es que en nuestro país ese décalage, que se concreta en la posibilidad de que un partido que no supera el 50\% de los votos obtenga una mayoría absoluta sólida en el Congreso de los Diputados, se justifica en la cohesión territorial y en la consiguiente asignación de un número mínimo de escaños a cada provincia; previsión que se contiene, en parte, en la propia Constitución, y que ha resultado más eficaz para consolidar mayorías estables que el propio sistema electoral D'Hont.

Mientras que, en la ley electoral italiana, el beneficio en escaños a favor de la formación política más votada se deriva de una previsión normativa ideada en la teoría para la estabilidad gubernamental, pero ineficaz en la práctica para garantizarla, como se ha dicho. Por esta razón, la doctrina ha vinculado esta previsión del premio di magggioranza, más bien, con las leyes electorales aprobadas en Francia y en Italia en los años 50 para reducir o eliminar la representación parlamentaria del Partido Comunista, leyes a las que se calificó en aquel momento de "loi scélerate" (ley perversa, en Francia) y de "legge truffa" (ley estafa, en Italia).

Queda por señalar, en cuanto al sistema de reparto de escaños para la Cámara de Diputados, que los asignados a cada coalición a nivel nacional se reparten según el sistema del resto mayor entre los partidos coaligados que superan las barreras legales pertinentes; posteriormente, los escaños que corresponden a cada partido se reparten entre las listas del mismo presentadas en cada circunscripción electoral (provincia), de acuerdo con los votos obtenidos en cada una de ellas, determinándose después, a partir de esas listas provinciales de candidatos y del orden que éstos ocupan en las mismas, las personas que han de ser proclamadas electas como diputados.

La falta de razonabilidad del premio de mayoría a la que se ha hecho referencia se acentúa, si cabe, en el sistema electoral del Senado. Aquí la circunscripción electoral es la región, pero el premio de mayoría y el reparto de escaños a través del sistema proporcional del resto mayor se establecen también en ese ámbito territorial.

De esta forma, entran en el reparto de escaños en cada región las coaliciones que hayan superado el $20 \%$ de los votos en la circunscripción, siempre que la lista de uno de los partidos coaligados alcance al menos el 3\% de los votos válidos en la misma. En cuanto a la barrera legal que se exige a los partidos, entrarán en el reparto de escaños aquéllos integrados en una coalición que supera la anterior barrera legal, siempre que alcancen el 3\% de los votos; y también los partidos no coaligados, o integrantes de una coalición que no supera la barrera legal del 20\%, siempre que hayan obtenido al menos el $8 \%$ de los votos regionales. Por último, el premio de mayoría para el partido o coalición más 
votado conlleva que se le atribuyan el 55\% de los escaños asignados a la región, salvo que por su número de votos y aplicando la fórmula electoral del resto mayor le correspondiesen más.

La primera disfuncionalidad de esta regulación legal deriva de las distintas barreras legales que se exigen a los partidos para poder obtener escaño, ya se encuentren coaligados (3\%) o no (8\%), y de la falta de justificación de este trato diferenciador, en los términos que se han explicado. Además, al contrario de lo previsto en el sistema de la Cámara de Diputados, para la elección del Senado no se prevé ningún supuesto en el que un partido que no supera la barrera legal se incorpore al reparto de escaños; pudiéndose producir la paradoja, por ejemplo, de que una coalición supere el 20\% de los votos pero sólo uno de los partidos que la integran alcance el 3\% de los sufragios, situación en la cual corresponderían a ese único partido todos los escaños asignados a la coalición en la región e incluso, si su coalición fuese la más votada, el premio de mayoría (con el 3\% de los votos podría obtener el 55\% de los escaños).

Esta falta de razonabilidad del sistema electoral del Senado se deriva, a su vez, del ámbito territorial donde se produce la asignación de escaños. Ello podría traer como consecuencia que se constituyan coaliciones coyunturales en cada una de las regiones, en función de las fuerzas políticas presentes en las mismas y de las posibilidades de obtener el mayor número de votos y el correspondiente premio de mayoria, con lo que no se garantiza la existencia de una coalición mayoritaria sólida a nivel estatal. Por otra parte, al atribuirse este premio en cada región y en atención a los resultados electorales que se dan en la misma, puede ocurrir que este mecanismo sea inoperante para garantizar una mayoría de gobierno en el Senado porque se compensen los premios que reciben las distintas coaliciones en cada una de las circunscripciones.

Pero las merecidas críticas al nuevo sistema electoral no concluyen ahí. Así, la ley n. ${ }^{\circ} 270$ de 2005 prevé para las elecciones a la Cámara y al Senado que las listas sean cerradas y bloqueadas, reduciéndose considerablemente la capacidad de elección de los ciudadanos y asumiendo los partidos una capacidad de decisión amplísima a la hora de determinar los candidatos con posibilidades de resultar electos. Estas características de las listas presente también de forma bastante generalizada en nuestro ordenamiento, donde no faltan críticas en el sentido referido, es difícilmente aceptable en el sistema italiano, puesto que allí el sistema electoral proporcional ha venido acompañado siempre de la facultad de los ciudadanos para dar un voto de preferencia a candidatos de las listas presentadas por los partidos.

Además, la doctrina ha considerado que la ley electoral incurre en una flagrante inconstitucionalidad por omisión, al no prever ningún mecanismo para promover en los procesos electorales la paridad entre sexos que viene exigida por el art. 51 de la Constitución [«... todos los ciudadanos de uno y otro sexo pueden acceder a las funciones y a los cargos públicos electivos en condiciones de igualdad, según los requisitos establecidos en la ley... a tal fin la República promueve con disposiciones específicas (appositi provvedimenti) la paridad de oportunidades entre el hombre y la mujer.]; más aún cuando la propia Corte 
constitucional ha afirmado que del citado precepto se deriva "una actuación promocional obligada (doverosa) para la paridad de acceso en las elecciones" (sentencia n. ${ }^{\circ} 49$ de 2003).

\section{EPPUR SI MUOVE...}

Otro bito fundamental en la vida constitucional italiana del último año ha sido la aprobación parlamentaria de una profunda reforma de la parte orgánica de la Constitución, que no ha entrado finalmente en vigor al no haber sido ratificada por el cuerpo electoral en referéndum. Alguno de los contenidos de esta reforma constitucional se reseñaban en la anterior crónica (los referidos a la organización de las competencias regionales), aunque la sensatez del cuerpo electoral que ha votado negativamente sobre la misma nos exime de dar cumplida cuenta, ahora, del resto de esos contenidos. Sólo nos detendremos, brevemente, en algunas pinceladas que ponen de manifiesto la intrínseca falta de racionalidad de la propuesta.

Los titulares de impacto con los que se ha presentado esta reforma de la Constitución preparada por la mayoría de gobierno de la pasada legislatura (Polo delle Libertà), que no contó con el apoyo de la oposición ahora en el gobierno (L'Unione), se han referido a la misma como la reforma del federalismo o, en terminología de los confederales de la Lega Nord aliados del Polo, como la reforma de la devolution. Sin embargo, esta propuesta de modificación constitucional era mucho menos que una redefinición del modelo territorial italiano en clave federal, y era mucho más que una simple alteración del reparto de competencias entre el Estado y las regiones.

Desde el primer punto de vista, la reforma introducía las competencias exclusivas regionales (asistencia y organización sanitaria; organización de la enseñanza, gestión de los centros educativos y formativos, a salvo la autonomía de los centros escolares; la definición de los programas educativos y formativos de interés específico de las regiones; la policía administrativa regional y local; y una cláusula residual, cualquier otra materia no reservada expresamente a la legislación del Estado). En cualquier caso, bajo la perspectiva de un Estado profundamente descentralizado como el español, considerar una propuesta de reforma como federal en razón de esas competencias atribuidas a las regiones, aunque se haga a título exclusivo, parece bastante exagerado.

Y más si tenemos en cuenta que, tras la reforma, el Estado seguiría disponiendo de competencias exclusivas en ámbitos materiales estrechamente vinculados con los anteriores (normas generales sobre la tutela de la salud; seguridad y calidad alimentaria; normas generales sobre la educación), o que subsistirían ámbitos materiales concurrentes (donde al Estado le corresponde aprobar las normas básicas al respecto) también muy interrelacionados con esas supuestas competencias exclusivas regionales (como por ejemplo alimentación; o educación). 
Pero aún más, la reforma añadía nuevas competencias exclusivas del Estado, o redefinía como tales materias que eran concurrentes en la reforma de 2001 (hasta 13 nuevos ámbitos materiales exclusivos se asignaban a aquél); reintroducía el control político del Estado (a través del Parlamento en sesión conjunta) sobre las leyes regionales para preservar el interés nacional (control eliminado en la reforma de 2001); incorporaba nuevos poderes sustitutivos del Estado frente a los incumplimientos de las regiones (incluida la sustitución legislativa); y eliminaba la cláusula introducida en el art. 116 de la Constitución italiana en la reforma de 2001, cláusula que habilita la posibilidad de un reparto competencial asimétrico entre las distintas regiones (regionalismo a geometria vairabile).

Parece claro que todas estas previsiones contenidas en la reforma tendían más bien hacia una redefinición de las competencias en clave centralizadora, algo muy distinto al espiritu que se achacaba a la propuesta. En definitiva, como escribíamos en la crónica anterior, esta propuesta de modificación de la Constitución parecía pensada más bien para poner palos en el rodaje del modelo regional redefinido tras la reforma de 2001; modelo al que no ponen demasiados peros algunos líderes del centro-derecha de regiones con un impulso autonomista (que no secesionista) muy fuerte, como puede ser la Lombardía (gobernada por Forza Italia), regiones que disponen ahora de la capacidad de alcanzar un nivel de autogobierno no muy distinto al que se atribuyó en el origen de la Constitución republicana a las regiones de estatuto especial. Por eso, en el momento en que Italia parece moverse hacia un Estado auténticamente descentralizado, la propuesta constitucional de 2006 parecía querer hacer retornar el sistema de poder a su tradicional estanquidad.

Pero lo más reseñable es que, tras la cortina de humo de la devolution, la reforma constitucional rechazada en el referéndum reescribía prácticamente en su totalidad la forma de gobierno del país, empezando por los poderes del Presidente de la República, pasando por la forma de designación de la Corte constitucional y sus competencias, y cerrando con la definición de nuevos poderes para el jefe del ejecutivo en su relación con el Parlamento. Aunque de nuevo aquí la propuesta pecaba de incoherencia interna.

Basta para demostrar esta afirmación con poner de manifiesto el modo en que se organizaba la designación del jefe de gobierno en la reforma constitucional fallida. De partida, en ésta se pretendía fortalecer los poderes del que se denominaba ya no Presidente del Consiglio sino Primo Ministro, para permitirle mantener cohesionada la mayoría de gobierno. Se le asignaba, por ejemplo, la facultad de disolver anticipadamente la Cámara de Diputados o la de designar y remover libremente a los ministros.

Al mismo tiempo, la propuesta pretendía garantizar una legitimación democrática directa del Primer Ministro; para ello, preveía la necesaria vinculación de las diferentes candidaturas a la Cámara de Diputados con los candidatos a dicha magistratura, al modo en que se establece en la ley electoral a la que hemos dedicado el apartado anterior. Como complemento a este presupuesto normativo, la reforma establecía la exigencia de que la ley electoral favoreciese la 
creación de una mayoría parlamentaria vinculada al candidato al puesto de Primer Ministro (lo que legitimaría constitucionalmente, aquí sí, el premio de mayoría). Además, se disponía expresamente que el Presidente de la República debía nombrar al Primer Ministro tomando como base los resultados de las elecciones a la Cámara de Diputados.

Por otra parte, la reforma avanzaba hacia la consolidación de una forma de parlamentarismo racionalizado al establecer que la relación de confianza se instauraría únicamente entre la Cámara de Diputados y el Primer Ministro, saliendo el Senado llamado "federal" del circuito del otorgamiento y remoción de la fiducia parlamentaria al Gobierno. De esta forma, aunque el Primer Ministro nombrado por el Presidente de la República debía presentar su programa de legislatura y la composición de su gobierno en ambas Cámaras, sólo se preveía el voto sobre el programa en la Cámara de Diputados, como ratificación parlamentaria del nombramiento predeterminado por los ciudadanos en las elecciones.

Pero aquí concluyen los instrumentos previstos en la propuesta de reforma constitucional para racionalizar la forma parlamentaria de gobierno; además, otras instituciones contenidas en la misma echaban por tierra buena parte de la virtualidad práctica de esos instrumentos para preservar la estabilidad del gobierno, o para definir una forma política auténticamente parlamentaria. Por ejemplo, aunque el Presidente de la República venía obligado a disolver la Cámara de Diputados cuando se lo solicitara el Primer Ministro, que asumía, así, la entera responsabilidad de la decisión, dicha disolución no se llevaba a efecto si la mayoría parlamentaria derivada de las elecciones que supusiera, al mismo tiempo, la mayoría de los componentes de la Cámara, decidía continuar con el programa de la coalición nombrando un nuevo Primer Ministro en el plazo de 20 días.

En otras palabras, se trataba de un poder de disolución disminuido, eficaz sólo si no se daban nuevas componendas en el seno de la mayoría que concluyeran en la designación de un nuevo Primer Ministro (hipótesis nada desdeñable en los gobiernos de coalición multipartidista que se dan en Italia, como ocurrió por ejemplo en 1998 con la caída de Romano Prodi y el nombramiento de Máximo D'Alema como Presidente del Consejo de Ministros).

Esa atemperación de la forma de gobierno de canciller se derivaba, además, de la regulación que hacía la propuesta constitucional de cuatro situaciones diferentes a través de las cuales se podía medir la confianza de la Cámara de Diputados hacia el Primer Ministro. En primer término, allí se preveía la posibilidad de que éste plantease una cuestión de confianza sobre propuestas del gobierno (salvo sobre las leyes constitucionales y las leyes de reforma de la Constitución); en este caso el Primer Ministro se encontraba supeditado a la cohesión de su mayoría parlamentaria y, en último extremo, al chantaje (ricatto) de los pequeños partidos integrantes de la coalición de gobierno (la minoranza della maggioranza) que, votando con la oposición, podían alterar el reparto de fuerzas en la Cámara.

Lo mismo que ocurriría en el caso de la moción de censura tout court que se disciplinaba en la propuesta de modificación del artículo 94, a través de la 
cual la mayoría absoluta de la Cámara de Diputados (sin ninguna limitación en cuanto a los partidos cuyos votos configurarían esa mayoría, pudiendo provenir de parte o de toda la oposición y de parte o de toda la mayoría derivada de las elecciones), podía provocar la dimisión del Primer Ministro, aunque sin la posibilidad de nombrar otro distinto, puesto que la consecuencia necesaria de esta moción era la disolución de la Cámara y la convocatoria de elecciones.

Aún más, en el supuesto de que esta moción de censura no prosperase como consecuencia del voto negativo a la misma de la oposición (mozione di sfiducia... respinta con il voto determinante di deputati non appartenenti alla maggioranza espressa dalle elezioni), el Primer Ministro venía obligado también a dimitir; aunque aquí la convocatoria de elecciones no era automática, ya que la mayoría derivada de las elecciones que constituyera, al mismo tiempo, la mayoría parlamentaria, podía optar por proponer y nombrar un nuevo Primer Ministro (posibilidad que también se preveía para el caso de que no se aprobara una cuestión de confianza planteada por el Primer Ministro).

Para concluir, la mayoría derivada de las elecciones que constituyera la mayoría de la Cámara podía plantear, también, una moción de censura constructiva frente al Primer Ministro, proponiendo y designando un nuevo candidato a ese cargo. En definitiva, los reequilibrios en el seno de la coalición mayoritaria de gobierno podían derivar también en la designación de un nuevo jefe del ejecutivo.

Si se observa bien, eran diversos los supuestos contenidos en la propuesta de reforma constitucional (moción de censura constructiva, moción de censura que no prospera por el voto de la oposición, cuestión de confianza que no prospera) en los que se rompía la vinculación entre la mayoría parlamentaria derivada de las elecciones y el candidato al cargo de Primer Ministro acerca del que se habían pronunciado expresamente los electores. Dicho de otra forma, estos mecanismos seguían situando al jefe del ejecutivo bajo la dependencia de los acuerdos de coalición haciendo menos fácil su liderazgo; seguían presuponiendo, en definitiva, que son los partidos los que tienen la llave de la estabilidad del gobierno, que son éstos los que dirigen al ejecutivo y no el Primer Ministro el que dirige, cohesiona y lidera al gobierno y a su mayoría parlamentaria.

De la propuesta de reforma constitucional podría seguirse escribiendo, de modo crítico, durante muchas más páginas, por ejemplo haciendo referencia a la configuración que se hace del Senado federal y del procedimiento legislativo en la misma ${ }^{2}$; pero no podemos olvidar que es una reforma fracasada y que los últimos meses han supuesto un cambio de rumbo en la política italiana que abre nuevos retos para el fututo constitucional.

Así se ha puesto de manifiesto por diversos autores en sede doctrinal (A. Barbera, S. Ceccanti, A. Pace, V. Onida, entre otros), que han señalado las ne-

2 Sobre estas cuestiones, permítase el reenvío a ORTEGA SANTIAGO, C., "La reforma constitucional del Estado regional italiano", en Revista Española de Derecho Constitucional, n. ${ }^{\circ}$ 75, 2005, p. 181 y ss., donde se explica por qué el Senado previsto en la propuesta de reforma no puede entenderse como un Senado auténticamente federal. 
cesidades de reforma constitucional (parcial) aún presentes en el texto italiano. A grandes rasgos, se ha apuntado la necesidad de redefinir el reparto de las competencias derivado de la reforma de 2001, asegurando la autoridad del Estado y su preeminencia en determinados ámbitos, sin renunciar a una intensa descentralización política; y se ha señalado la urgencia por regular un Senado realmente representativo de las regiones (A. Barbera, S. Ceccanti).

Al mismo tiempo, como consecuencia de la instauración de un sistema político bipolar y de la configuración del Parlamento a partir de una clara división entre mayoría y oposición (final de la llamada democrazia consociativa), se haría necesario delimitar una serie de decisiones que deberían corresponder ya no a una simple mayoría, sino a mayorías cualificadas (legislación electoral, garantías de la oposición en sede parlamentaria - Reglamentos-, recurso ante la Corte constitucional frente a la verificación de poderes realizada por las Cámaras; A. Pace). Sin olvidar la necesidad de modificar de nuevo la ley electoral, haciéndola retornar a la lógica del sistema mayoritario y compadeciéndola con la Constitución.

Por último, como ya señalábamos en la crónica anterior, lo que se precisa actualmente en el ordenamiento italiano, más que reformas constitucionales intensas y extensas en el ámbito territorial, es la plena actuación del diseño constitucional sobre la cuestión. Un buen ejemplo se encuentra en el ámbito del ejercicio de la potestad tributaria por los entes regionales y locales, que no puede llevarse a cabo a falta de una disciplina legal estatal que defina el marco y los límites de dicho ejercicio (sentencia de la Corte constitucional n. ${ }^{\circ} 37$ de 2004). Algo parecido a lo que ocurre con la posibilidad recogida en la Constitución (art. 116) de que las regiones ordinarias accedan a formas de autonomía diferenciadas, lo cual permitiría alcanzar cierta especialidad competencial a regiones que en la actualidad tienen un fuerte impulso autonomista (Lombardía, Véneto) pero que no han sido configuradas como regiones especiales en la Constitución (V. Onida).

Que las reformas institucionales deben moverse por ese camino más sensato, parece demostrarse en los resultados del referéndum de junio sobre la propuesta de reforma constitucional de la que hemos venido hablando. Un $61,3 \%$ de los electores se manifestaron en contra de la misma, triunfando el No tanto en el Norte del país (52,6\%), como en el Centro (67,7\%); en el Sur $(74,8 \%)$ y en las Islas $(70,6 \%)$; produciéndose la victoria del Sí sólo en dos regiones, Lombardía $(54,6 \%)$ y Véneto $(55,3 \%)$.

Las reflexiones de una autoridad como el profesor Zagrebelsky, realizadas hace ya algunos años, quizá sean un buen contrapunto para adivinar cuáles son los momentos y los modos más adecuados para llevar a cabo una reforma constitucional en Italia, evitando así al cuerpo electoral la gravosa decisión sobre propuestas de modificación heterogéneas y sin coherencia interna: «La exigencia de la reforma nace de la disgregación del proceso de integración política; pero la reforma constitucional presupone ella misma el máximo de agregación. Se quiere la reforma porque no se consigue decidir; pero la reforma de la Constitución (en el sentido hipotizado) es ella misma la máxima decisión hi- 
potizable. Cuanto mayor es la disgregación, mayor es la necesidad de la reforma; pero cuanto más necesaria es ésta, es más difícil. Hasta el límite de la paradoja: cuando la reforma es absolutamente indispensable, entonces es también absolutamente imposible" (G. ZAGREBELSKY, "Adeguamenti e cambiamenti della Costituzione", en Scritti in onore di Vezio Crisafulli, vol. II, Cedam, Padua, 1985, p. 915).

ABSTRACT. In the last months the italian cytizens had questioned with their votes in the general elections and in the referendum, two important initiatives of Berlusconi's Government, the electoral reform and the proposal of the constitutional reform. The first had been confined in a law very criticized by wide sectors of italian constitucional doctrine because she designes an electoral system that lacks of reassonability between the aims she try to get and the measures to get them. The proportional electoral system, the legal barriers, the prize of majority, the omission of measurements to obtain the parity among men and women in the access of representative charges, they are all difficulty reconciliable contents of the law with the inforce Constitution. The coalition "Casa delle Liberta" has worked out damaged for losing the election under this electoral legislation and also for the rejection of citizens to bis offer of constitucional reform. A reform that was not a federal reform but a centralized reform. A reform that tried to introduce a chancellor government, but it was only an intention, because she had also institutions as the censure motions that permited the dominant position of the political parties. 